\title{
Using One-Dimensional Finite Element Analysis to Estimate Differential Pressure of Renal Artery Stenoses
}

\author{
BN Steele
}

North Carolina State University, Raleigh, NC, USA

\begin{abstract}
A study was conducted to evaluate the ability of a simple one-dimensional (1D) computational fluid dynamics (CFD) model to identify a hemodynamically significant renal artery stenosis by predicting pressure loss and flow rate across a renal artery stenosis. Six combinations of wall properties, inlet, and outlet boundary conditions were used to evaluate their effects on blood pressure estimation. All combinations of boundary conditions in the $1 D$ model produced pressure to flow relations that compared favourably with previously reported in vitro and three-dimensional CFD model of a similar geometry. The mean error of the $1 D$ results, using the in vitro model as the gold standard, ranges from 0.53 to $3.46 \mathrm{mmHg}$. While further work is required to optimize the specification of renal outlet boundary conditions from patient specific data, these results show that a $1 D$ model may be used to identify pressure gradients across a renal artery stenoses.
\end{abstract}

\section{Introduction}

A hemodynamically significant renal artery stenosis (RAS) can lead to hypertension, renal failure (ischemic nephropathy), refractory angina, and/or recurrent episodes of congestive heart failure and flash pulmonary edema. The definitive diagnosis of RAS is somewhat controversial in that the presence of anatomic RAS does not necessarily imply that hypertension or renal failure are caused by the RAS. Therefore, while non invasive imaging studies are used to identify lesions, pressure gradients should be obtained to confirm the hemodynamic significnce of a given stenosis.

Hemodynamic significance is generally accepted as a peak systolic pressure gradient of more than $10 \mathrm{mmHg}$ or a mean pressure gradient of more than $5 \mathrm{mmHg}$ across a stenosis [1]. Pressure gradients are evaluated using invasive catheterization, which exposes the patient to risk. In an effort to redice the risk assosciated with invasive pressure measurement of RAS, we present a method to numerically predict the pressure gradient across a stenosis. This method was originally proposed by Yim et al. [2] with the use of three-dimensional (3D) CFD method. Yim used an in vitro model and corresponding 3D CFD analysis to measure and predict the pressure gradients associated with varying levels of flow through a renal artery branch (Fig 1). We will show that the 1D method produces equivalent results and highlight the effect of model parameters on the solutions.

\section{Methods}

We have previously developed an integrated system for describing and solving flow, pressure, and area through a network of distensible tubes [3-5]. Breifly, this system solves axisymmetric $1 \mathrm{D}$ equations for flow $q$, and pressure $p$ :

$$
\begin{aligned}
& \frac{\partial s}{\partial t}+\frac{\partial q}{\partial z}=0 \\
& \frac{\partial q}{\partial t}+\frac{\partial}{\partial z}\left(\frac{4}{3} \frac{q^{2}}{s}\right)+\frac{s}{\rho} \frac{\partial p}{\partial z}=-N \frac{q}{s}
\end{aligned}
$$

and a constitutive equation relating pressure and area, $s$ :

$$
\begin{aligned}
& p(s(z, t), z, t)=p_{0}+\frac{4}{3} \frac{E h}{r_{0}(z)}\left(1-\sqrt{\frac{s_{0}(z)}{s(z, t)}}\right) \\
& \frac{E h}{r_{0}(z)}=k_{1} e^{-k_{2} r_{0}(z)}+k_{3} .
\end{aligned}
$$

The constitutive equation above is a first order elastic function with $k_{1}, k_{2}$, and $k_{3}$ fit to experimental data [6].

Because the 1D method assumes axial flow dominates, it neglects secondary flows. However, these secondary flows lead to significant losses in geometrically complex regions including stenosis and junctions. These losses are included in the $1 \mathrm{D}$ method via a minor loss coefficient, $K$, that is implemented in the viscous loss term in Equation 2 by $N=q K / 2 l$.

The stenosis loss model (Equation 5) used in this system was developed by Seeley and Young [7]. Here subscript 0 implies terms in the unobstructed, downstream region and 1 in the stenosed region. $R e$ is the Reynolds number, $K_{t}$ is an approximation that represents the turbulent effects, and $K_{v}$ represents the viscous effects. This model was developed using both 
concentric and eccentric geometries.

$$
\begin{aligned}
K & =2\left(\frac{K_{v}}{R e_{0}}+\frac{K_{t}}{2}\left[\frac{s_{0}}{s_{1}}-1\right]^{2}\right)\left(\frac{s_{1}}{s_{0}}\right)^{2} \\
K_{t} & =1.52 \\
K_{v} & =32 \frac{l}{2 r_{0}}\left(\frac{s_{0}}{s_{1}}\right)^{2}
\end{aligned}
$$

While the flow fields produced by an eccentric and concentric stenoses would differ, the overall pressure loss is not affected. In general, stenosis are not considered hemodynamically significant until there is a $75 \%$ reduction in area. This generalization may vary for a given artery based on a number of physiologic factors including length, resistance of the distal bed, and collateral flow and not on the hemodynamics of the geometry in particular.

The model geometry used in this study was adapted from Yim et al.[2] Fig 1. The diameter of the aorta and renal artery are specified as $2.43 \mathrm{~cm}$ and $0.57 \mathrm{~cm}$ respectively. Observation of the model shows that a $70 \%$ stenosis was used. Our model was scaled slightly to accommodate renal outlet boundary conditions as discussed below. The relevant renal artery diameters used are $0.5 \mathrm{~cm}$ with a minimum stenosis of $0.27 \mathrm{~cm}$ and a length of $0.705 \mathrm{~cm}$. After the renal artery junction, the aorta was tapered to an outlet diameter of $1.0 \mathrm{~cm}$.

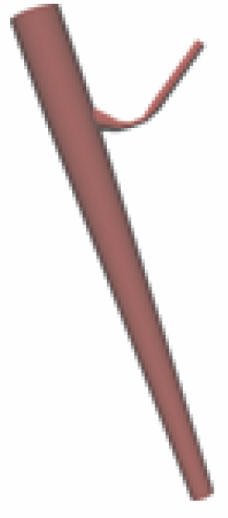

Figure 1. Renal steosis model
Inlet Waveform

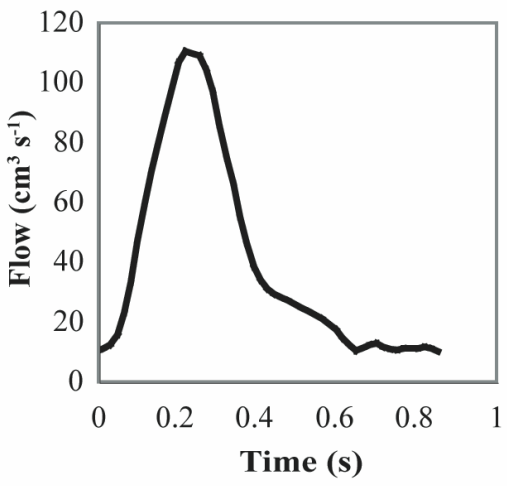

Figure 2. Inlet aortic flow waveform
Six combinations of boundary conditions were used to determine their impact on the predictive ability of the 1D model. The variable boundary conditions are inlet, outlet, and wall properties. Because pressure gradient is the only quantity reported, a steady inlet flow is sufficient to drive the analysis. However, the effects of pulsatile flow on pressure gradient are of interest. All studies were run with a mean inlet flow of $40 \mathrm{~cm}^{3} \cdot \mathrm{s}^{-1}$, with the pulsatile studies using the waveform shown in Figure 2. As discussed previously, an elastic wall property related to vessel radius in Equation 4 is used in the 1D code. A rigid wall approximation was achieved by specifying a constant modulus of $E h / r_{0}=1.99 \cdot 10^{7} \mathrm{~g} \cdot \mathrm{s}^{-2} \cdot \mathrm{cm}^{-1}$, the maximum value obtained using Equation 4.

The renal outlet boundary condition was varied to moderate renal artery flow. Impedance boundary conditions based on structured trees [5] and equivalent resistance boundary conditions (i.e. zero frequency impedance) were used. Briefly, the structured tree is an asymmetric fractal-like tree that is used to compute impedance based on linearized, axisymmetric equations for 1D flow [6]. The structured tree is adjusted by specifying the root radius, $r_{0}$, (the outlet of the modelled region) and a length-to-radius ratio, $l_{\mathrm{rr}}$. The structured tree can be modified using a scaling factor, $f$, on the "resistance" vessels $(\mathrm{r}<250 \mu \mathrm{m})$ to simulate autoregulation. The assignment of outlet boundary conditions will ultimately dictate the distribution of flow through a network of vessels and care must be taken to specify reasonable values. Representative impedance boundary conditions were previously determined based on average physiological flow distributions in a healthy adult. These values were used to specify the baseline impedance boundary condition as $r_{0}=0.5 \mathrm{~cm}, l_{r r}=80$ and $f=1.0$ for the aorta and $r_{0}=0.25, l_{r r}=21$ and $f=1.0$ for the renal artery [5]. In order to determine a range of flow and pressure gradients across the RAS, scaling factors $f=\{.6, .7, .8,1,1.1,1.2,1.3,1.5,2,2.5\}$ were used to increase or decrease the impedance to flow through the renal artery.

In preliminary studies, analyses were run with and without the stenosis loss model. It was determined that even though the $70 \%$ stenosis used would not necessarily meet the clinical definition of significance, the loss model was required to produce results of similar magnitude to the in vitro data. The minor loss model was more sensitive to changes in flow rate, with a higher slope between predicted pressure gradient and flow, than the in vitro model. In the loss model (Equation 5), the only term approximated based on experimental data was the turbulence loss coefficient $K_{t}=1.52$. We "tuned" the stenosis loss model by setting $K_{t}=1.20$ to reduce the slope between pressure gradient and flow. This tuning is only appropriate for cases where training data is available.

\section{Results}

Studies were performed to evaluate the ability of the 1D model to predict flow and pressure drops across a RAS. Studies were performed using steady or a pulsatile inlet (aortic) flow waveform in both rigid and deformable tubes. At the renal outlet, impedance or the equivalent resistance boundary condition was used. At the aortic 
outlet, a consistent impedance boundary condition was used for all models. An overview of the effects of the different models and boundary conditions are shown in Figure 2.

The renal outlet boundary condition was adjusted to vary the flow through the renal artery. At a baseline level of impedance with scaling factor $f=1.0$, mean flow through the renal artery is between $10-13 \mathrm{~cm}^{3} \cdot \mathrm{s}^{-1}$, with pressure gradient ranging from 14-20 $\mathrm{mmHg}$ (Figure 3). By reducing the renal impedance, additional flow was drawn through the renal artery with an accompanying increase in pressure gradient across the stenosis. Increasing the renal impedance reduced the flow and pressure gradient across the stenosis. To compare the results with those published by Yim et al., the mean pressure gradient (renal pressure minus aortic pressure) and flow were computed for each model and scaling factor. The results of these studies along with the results of Yim's in vitro study and 3D CFD analysis are shown in Figure 3.

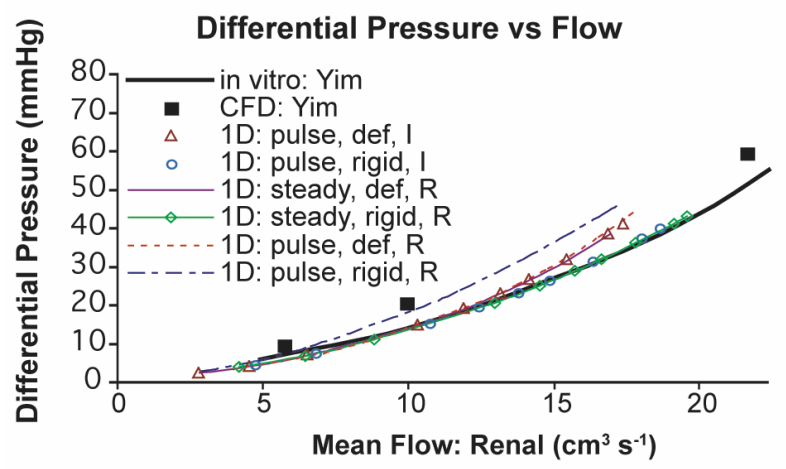

Figure 3. Comparison of $1 \mathrm{D}$ results(various) with in vitro (solid black) and 3D (solid Square). Pressure gradients reported with corresponding flow through renal artery.

For the purposes of this study, the values measured from the in vitro model are considered the "gold standard". Aortic pressures were not reported, therefore only mean gradient values can be compared.

\section{Discussion and conclusions}

To validate the utility of the $1 \mathrm{D}$ analysis method in predicting pressure gradients, a comparison was made against in vitro and CFD data reported by Yim et al. Yim was able to show agreement between the in vitro and CFD results (error $5.5 \mathrm{mmHg}$ ). We repeated the computational study using our 1D analysis method. The 1D model produced differential pressure vs. flow values similar to those measured in vitro through a RAS (Figure 3).

The 1D analyses examined a variety of boundary condition combinations. Overall, the 1D results were exceptionally close to the reported in vitro results. At higher flow rates, computed gradients were overestimated by most of the computational methods. There was little difference between the deformable models regardless of inlet or renal boundary conditions. The mean error between the deformable models and the in vitro model in the region of relevant pressures is $\sim 0.99 \mathrm{mmHg}$ with the greatest error occurring above a gradient of $12 \mathrm{mmHg}$. The deformable models exhibit a greater increase in differential pressure with flow than the rigid models. This is most likely because the variable elasticity allows distension of the aorta without allowing distension of the RAS, despite the fact that the absolute pressures decrease as pressure gradient increased. Similarities were also seen between two of the rigid models: steady flow with resistance and pulsatile flow with impedance boundary conditions. This rigid pair produced results closest the in vitro model over a large range of pressure gradients with a mean error of $\sim 0.5 \mathrm{mmHg}$. This agreement is expected since these boundary conditions are most similar to the experimental setup. Finally, the rigid walled model with pulsatile flow and resistance boundary conditions exhibits a mean error of $3.46 \mathrm{mmHg}$ and exhibited a trend closest to the reported $3 \mathrm{D}$ CFD values over a large range of pressures.

In this study, we find that the 1D analysis method using the stenosis loss model is very good at predicting pressure gradients when compared with in vitro experimental data. What remains to be determined is if this method can provide meaningful data to clinicians in predicting the benefits or outcome in treating RAS. The most meaningful data is likely to be the predicted waveform distal to the stenosed segment, the RPP. In order to predict an accurate RPP, more attention must be paid in modelling the outlet boundary condition for the renal artery and in realistic representation of vessel wall properties including both diseased and healthy tissues. While all 1D models produced reasonable pressure gradients, Figure 4 shows that the three boundary condition cases most likely to predict accurate waveforms are the rigid model with impedance boundary conditions or the deformable models with resistance or impedance boundary conditions. In an in vivo setting, an impedance boundary condition would produce the most realistic results. The future direction for this work is to determine a mapping between patient specific renal parameters for use in determining impedance boundary condition parameters. Data regarding renal function will also be used to determine the dynamic regulation limits of the kidney. This ability to determine kidney function may allow for prediction of patient outcomes following revascularization. 


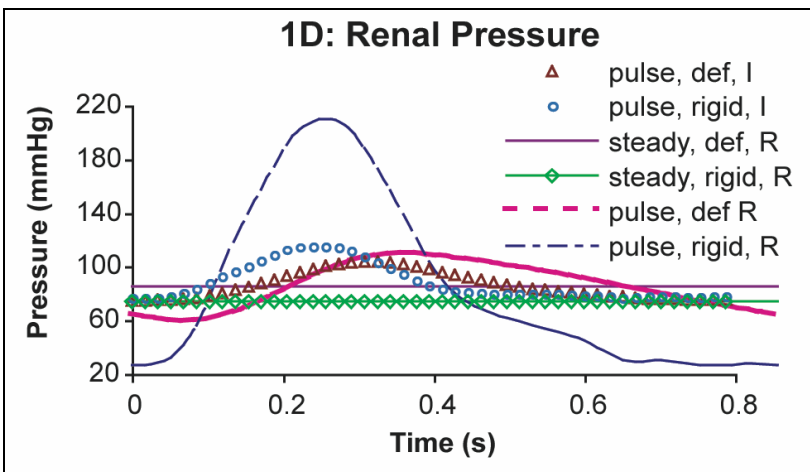

Figure 4. Computed flow waveform through renal artery. Boundary condition $(f=1)$ has great impact on flow waveform. Impedance boundary conditions produce most realistic values.

In addition to optimizing the renal artery boundary conditions, future work must address the feedback mechanisms regarding systemic boundary conditions. In order to report a meaningful pressure gradient as a percentage of aortic pressure, the computed aortic pressure must be correct. In this study, the outlet boundary condition for the aorta was not modified. While the baseline aortic pressures for outlet impedance boundary conditions with a scaling factor $f=1$ were approximately $100 \mathrm{mmHg}$, the system was not tuned to achieve physiologic pressures for scaled renal outlets. As a result, the predicted systemic pressures were widely varied with increased pressures for renal scaling factors $f<1$ and lowered pressures for scaling factors $f>1$. The scaling factors were selected to vary the flow through the renal artery and were not based on physiologic values. Therefore, it is clear that a better understanding of the limits of normal renal regulation and of the effects of renal flow and pressure on systemic regulation is needed to create an accurate computational model of blood flow and pressure through RAS.

We show that the $1 \mathrm{D}$ analysis method can predict a pressure gradient across a RAS with accuracy comparable to a 3D CFD model. The strength of a 1D method is the speed in which it can evaluate the pressure and flow distribution over a large region of interest. The limitation of a 1D method is that it cannot provide detailed flow field information. Therefore, the 1D method is viewed as a companion to $3 \mathrm{D}$ methods in assessing blood flow. The challenge for any analyst is to describe the region of interest with as much detail as needed to achieve a meaningful result. Imaging techniques are capable of providing geometry, and in some cases, flow of large vessels for model input. However, in order to accurately model the flow in both large and small vessels, more sophisticated boundary conditions are required.here

\section{Acknowledgements}

I would like to thank Dr. Richard Stouffer for his input regarding the treatment of renal artery stenosis.

\section{References}

[1] Sacks D, McClenny TE, Cardella JF, Lewis CA. Society of Interventional Radiology clinical practice guidelines. J.Vasc.Interv.Radiol. 2003 Sep;14(9 Pt 2):S199-202.

[2] Yim PJ, Cebral JR, Weaver A, Lutz RJ, Soto O, Vasbinder $\mathrm{GB}$, et al. Estimation of the differential pressure at renal artery stenoses. Magn Reson Med 2004 May;51(5):969-77.

[3] Wan J, Steele BN, Spicer SA, Strohband S, Feijoo GR, Hughes TJR, et al. A One-Dimensional Finite Element Method for Simulation-Based Medical Planning for Cardiovascular Disease. Comput Methods Biomech Biomed Engin 2002;5(3):195-206.

[4] Steele BN, Wan J, Ku JP, Hughes TJR, Taylor CA. In vivo Validation of a One-Dimensional Finite Element Method for Predicting Blood Flow in Cardiovascular Bypass Grafts. IEEE Trans Biomed Eng 2003 June;50(6):649-656.

[5] Steele BN, Olufsen MS, Taylor CA. Fractal network model for simulating abdominal and lower extremity blood flow during resting and exercise conditions. Comput Methods Biomech Biomed Engin 2007;10(1):39-51.

[6] Olufsen MS. A Structured Tree Outflow Condition for Blood Flow in the Larger Systemic Arteries. Am J Physiol 1999;276(1 Pt 2):H257-H268.

[7] Seeley BD, Young DF. Effect of geometry on pressure losses across models of arterial stenoses. J Biomech 1976;9(7):439-448.

Address for correspondence

Brooke N. Steele

2148 Burlington Labs, CB\#7115

North Carolina State University

Raleigh, Nc 27695-7115

bnsteel@ncsu.edu 